\title{
Estrutura e dinâmica do componente arbóreo em área de cabruca na região cacaueira do sul da Bahia, Brasil
}

\author{
Regina Helena Rosa Sambuichi ${ }^{1}$
}

Recebido em 22/07/2005. Aceito em 23/06/2006

\begin{abstract}
RESUMO - (Estrutura e dinâmica do componente arbóreo em área de cabruca na região cacaueira do sul da Bahia, Brasil). As cabrucas são áreas de cultivo onde o cacau foi implantado sob a sombra da floresta nativa raleada. Na região sul da Bahia, onde as florestas são poucas e fragmentadas, as cabrucas têm sido consideradas importantes para a conservação de espécies nativas. Visando avaliar a conservação a longo prazo de espécies arbóreas nativas nas cabrucas, foi realizado um levantamento fitossociológico das árvores sombreadoras em 1,7 ha de cabruca com cerca de 25 anos de implantação e avaliada a sua dinâmica após um período de sete anos. Foram encontrados 120 indivíduos e 62 espécies, com densidade média de 70,5 ind. ha ${ }^{-1}$, área basal média de $23,0 \mathrm{~m}^{2}$ ha ${ }^{-1}$ e diversidade de 3,88 nats ind. $^{-1}$ (Shannon). Após sete anos, houve redução de $8,4 \%$ na densidade, com taxa de mortalidade de $2,16 \%$ ano ${ }^{-1}$ e taxa de recrutamento de $0,81 \% \mathrm{ano}^{-1}$. A taxa média de incremento diamétrico foi de $0,7 \pm 0,6 \mathrm{~cm} \mathrm{ano}^{-1}$. A maioria das árvores pertenceu a espécies de florestas maduras, mas os novos indivíduos recrutados foram quase todos de espécies pioneiras. A estimativa de riqueza total (Chao) foi de 110 espécies, com redução de 20 espécies durante o período. Os resultados confirmaram as indicações de que as cabrucas representam um importante banco de espécies arbóreas nativas, mas estão sendo rapidamente alteradas, raleadas e empobrecidas. Isso alerta para a necessidade urgente de modificar a forma de manejo dessas áreas de maneira a permitir a conservação das espécies de florestas maduras. Os altos valores de área basal e de taxa de crescimento encontrados indicam a possibilidade de uso das cabrucas para o sequiestro e estoque de carbono.
\end{abstract}

Palavras-chave: Mata Atlântica, sistemas agroflorestais, cacau rústico, diversidade de espécies, conservação de espécies arbóreas, fitossociologia

\begin{abstract}
Structure and dynamics of the tree community in a "cabruca" area in the cacao region of southern Bahia State, Brazil). "Cabrucas" are areas where cacao trees are planted in the shade of thinned native forest. In southern Bahia State, where the forests are few and fragmented, cabrucas are important for native species conservation. Aiming to evaluate the conservation of native tree species in cabrucas on a long term basis, a phytosociological survey of the shade trees was carried out in a 1.7-ha area of cabruca ca. 25 years after implantation, and the dynamics were assessed after a period of seven years. One hundred and twenty trees were counted and 62 species identified. Mean density was 70.5 ind. ha ${ }^{-1}$, mean basal area was $23.0 \mathrm{~m}^{2} \mathrm{ha}^{-1}$ and diversity was 3.88 nats ind. ${ }^{-1}$ (Shannon). After seven years, there was an $8.4 \%$ reduction in density, with a mortality rate of $2.16 \%$ year ${ }^{-1}$ and a recruitment rate of $0.81 \%$ year ${ }^{-1}$. The mean increment in diameter was $0.7 \pm 0.6 \mathrm{~cm}_{\text {year }}{ }^{-1}$. The trees were mainly mature forest species, but newly recruited individuals were mostly pioneer species. Total estimated richness (Chao) was 110 species, with a reduction of 20 species during the period. The results confirmed indications that the cabrucas represent an important reservoir of native tree species, but these areas are quickly becoming altered, thinned and impoverished. There is an urgent need to modify current management practices in order to conserve mature forest species. The high basal area and growth rate indicate that the cabrucas may be used for carbon sequestration and storage.
\end{abstract}

Key words: Atlantic coastal forest, agroforestry systems, rustic cacao, species diversity, tree species conservation, phytosociology

\section{Introdução}

Em ecossistemas de alta diversidade biológica como as florestas pluviais tropicais, a maioria das espécies arbóreas, principalmente as de maior porte, apresenta baixa densidade de indivíduos, necessitando de grandes áreas para manter um tamanho mínimo viável de população (Bawa \& Ashton 1991). Em regiões onde a pressão antrópica é intensa, o estabelecimento e a manutenção de grandes áreas de reserva é muito difícil, principalmente quando as áreas de vegetação natural já se encontram muito reduzidas e fragmentadas. Nessas situações, uma alternativa a ser considerada é a conservação de espécies nativas dentro das áreas de cultivo, através de sistemas que aliem produção agrícola e conservação, servindo como área complementar e de apoio às reservas de vegetação nativa existentes (Pimentel et al. 1992; Schroth et al. 2004). Um sistema que vem despertando o interesse de pesquisadores e conservacionistas é o

1 Universidade Estadual de Santa Cruz, Departamento de Ciências Biológicas, Rodovia Ilhéus-Itabuna km 16, $45662-000$ Ilhéus, BA, Brasil (sambuich@uesc.br) 
cultivo sob o sombreamento natural da floresta (Perfecto et al. 1996; Johns 1999; Moguel \& Toledo 1999; Rice \& Greenberg 2000). Estudos realizados em áreas de cacau e café rústicos, plantados sob a floresta raleada, vem mostrando que essas áreas apresentam maior diversidade de fauna que áreas cultivadas sem sombra ou com sombreamento monoespecífico (Gallina et al. 1996; Greenberg et al. 1997; Perfecto et al. 1997; Johnson 2000; Reitsma et al. 2001; Perfecto et al. 2003; Sperber et al. 2004), principalmente se houverem áreas de florestas próximas ou adjacentes. A diversidade de espécies da fauna está também correlacionada com a densidade e diversidade da cobertura arbórea (Reitsma et al. 2001; Sperber et al. 2004).

A Floresta Atlântica é considerada uma das áreas prioritárias (hotspots) para conservação da biodiversidade no mundo (Myers et al. 2000). Uma de suas áreas mais ricas encontra-se na região sul do Estado da Bahia (Mori et al. 1983; Nobre 1998). Estudos mostram ser esta área uma zona de endemismo dentro da Floresta Atlântica (Thomas et al. 1998), apresentando também uma das maiores riquezas de espécies arbóreas do planeta (Thomas \& Carvalho 1997). Nessa região, a floresta se encontra atualmente reduzida a entre $1 \%$ e $10 \%$ da sua cobertura original, variando as estimativas de acordo com o grau de precisão destas em distinguir as áreas de floresta nativa preservada das áreas de floresta perturbada e das áreas com outros tipos de uso da terra (Saatchi et al. 2001).

Apesar de toda essa devastação, o sul da Bahia apresenta ainda uma significativa concentração de árvores nativas em relação a outras regiões da Floresta Atlântica devido ao sistema tradicional de plantio do cacau sob a sombra da floresta raleada, conhecido na região como "cabruca". Devido à atual crise econômica na cultura do cacau e à proibição à derrubada de florestas, desde o final da década de 80 praticamente não tem sido mais implantadas novas cabrucas na região. Estima-se, porém, que $70 \%$ dos $6.800 \mathrm{~km}^{2}$ de plantações de cacau existentes na região ainda esteja em sistema de cabruca (Araújo et al. 1998), o que representa uma área de cobertura considerável em comparação com a ínfima área de floresta nativa remanescente. No mosaico de paisagem formado pelas cabrucas, fragmentos de florestas e outras formas de uso da terra, as cabrucas formam uma matriz permeável, funcionando como corredores biológicos entre os fragmentos florestais e assim permitindo o fluxo genético entre populações, além de diminuir o impacto do efeito de borda sobre os fragmentos (Pardini 2004). Elas servem também como área complementar de forrageamento para espécies da fauna que vivem nesses fragmentos e nas áreas de reserva adjacentes (Dietz et al. 1996; Raboy et al. 2004). Um estudo realizado próximo à área da Reserva Biológica de Una mostrou que as cabrucas mantém pelo menos $70 \%$ das espécies de sapos terrestres, pequenos mamíferos e morcegos encontradas nas áreas vizinhas de floresta nativa (Pardini 2004).

Os levantamentos fitossociológicos feitos em cabruca (Sambuichi 2002; Rolim \& Chiarello 2004) indicaram que essas áreas podem se constituir em importantes bancos genéticos de espécies arbóreas nativas, porém, mostraram também que as espécies nativas de florestas primárias não estão sendo bem conservadas nessas áreas. Sambuichi (2002), levantando a vegetação arbórea de uma cabruca antiga no sul da Bahia, encontrou alto índice de diversidade, mas, constatou a invasão de árvores exóticas e secundárias, as quais se encontravam entre as espécies mais importantes na área. Rolim \& Chiarello (2004) encontraram uma significativa quantidade de espécies ocorrendo em cabrucas do Espírito Santo, mas observaram uma grande proporção de espécies de estágios iniciais de sucessão, alertando para a morte lenta das espécies de florestas maduras. Isso indica que as áreas de cabruca apresentam sérios problemas de regeneração natural, e que as espécies climáxcicas, características de florestas menos perturbadas, não estão conseguindo recrutar novos indivíduos nessas áreas. Indica também que as cabrucas estão se tornando, em composição florística, cada vez mais próximas de capoeiras e áreas degradadas, distanciando-se das florestas originais de onde foram em implantadas, causando um grande empobrecimento de espécies na região. Poucos trabalhos foram publicados ainda sobre o assunto, faltando embasamento científico para orientar as ações e decisões políticas que visam a conservação de espécies arbóreas na região. Pouco sabemos realmente sobre o que estamos perdendo nas cabrucas e a que velocidade isto está ocorrendo, e também sobre os fatores ambientais, principalmente relacionados ao manejo, que estão levando à mudança florística e consequiente perda de espécies nessas áreas. Este trabalho consiste no levantamento da vegetação arbórea de uma área de cabruca em dois momentos, avaliando a estrutura, a diversidade e a composição florística da vegetação remanescente da floresta nativa e a sua dinâmica por meio da avaliação da mortalidade e recrutamento de indivíduos, visando gerar subsídios 
para avaliar melhor o papel das cabrucas na conservação de espécies arbóreas nativas e embasar ações para tornar mais efetiva a conservação dessas espécies dentro do sistema agroflorestal de cabruca.

\section{Material e métodos}

Área de estudo - A área de estudo localiza-se na Fazenda Retiro, Município de Ilhéus, região sul do Estado da Bahia (14\%43'14" S, 3909'31' W). O clima da região é Af de Köppen. Chove na área em mais de 150 dias por ano, sendo que a precipitação pode chegar a $2.000 \mathrm{~mm}^{2} \mathrm{ano}^{-1}$. Não existe estação seca definida, podendo ocorrer, ocasionalmente, um a três meses com menos de $100 \mathrm{~mm}$ de chuva (Mori et al. 1983). A umidade relativa do ar, média anual, está entre 80-90\%. A temperatura média anual está entre $24-25^{\circ} \mathrm{C}$, com flutuação média anual entre $7-8{ }^{\circ} \mathrm{C}$, sendo os meses mais frios junho, julho e agosto. Encontra-se em área de domínio de argissolo vermelho-amarelo, ácido, de baixa a média fertilidade natural. As características químicas e físicas da camada de 0 a $20 \mathrm{~cm}$ do solo, em uma amostra composta de quatro sub-amostras coletadas aleatoriamente na área, são mostradas na Tab. 1. O relevo é ondulado.

$\mathrm{Na}$ região em estudo existem áreas de cabruca com diferentes idades de implantação. Devido a diferenças observadas na estrutura e na composição florística da vegetação, de acordo com R.H.R. Sambuichi (dados não publicados), estão sendo diferenciadas aqui as "cabrucas antigas", áreas estabelecidas desde o início do século XIX até meados do século XX, das “cabrucas novas", áreas implantadas no derradeiro ciclo de expansão da cultura entre as décadas de 60 a 80 , que ainda conservam principalmente as árvores originais da floresta. A vegetação da área de estudo é uma "cabruca nova", implantada entre os anos de 1973-1974. Na época foram selecionadas as árvores de maior porte e madeira mais resistente para servir de sombra ao cacau. Os cacaueiros foram plantados alinhados, com o espaçamento de $3 \times 3 \mathrm{~m}$. Desde sua implantação são mantidos na área tratos culturais como corte periódico da vegetação rasteira (roçagem), poda de ramos do cacaueiro, colheita de frutos, calagem e aplicação de fertilizantes, inseticidas e fungicidas. A cabruca estudada apresenta uma área total de aproximadamente 10 ha, circundada por cabrucas de outras idades, fragmentos de floresta, pastos e áreas de cacau com sombreamento monoespecífico.

Coleta e análise e dados - O primeiro censo foi realizado no ano de 1998. Foi demarcada uma área amostral de 1,7 ha subdividida em 34 parcelas de $20 \times 25 \mathrm{~m}$, dispostas em quatro faixas paralelas contíguas, duas de 11 parcelas, uma de sete parcelas e uma de cinco parcelas. A área apresentou um formato irregular porque foi demarcada de forma a incluir apenas as partes altas do terreno, evitando-se as encostas acentuadas e as partes baixas e alagadiças. Todos os indivíduos arbóreos, excluindo-se os cacaueiros, com $10 \mathrm{~cm}$ ou mais de DAP (diâmetro à altura do peito, a 1,30 $\mathrm{m}$ do solo) foram medidos, marcados com plaquetas de alumínio numeradas e identificados em nível de espécie ou morfoespécie. O DAP foi medido a partir do perímetro do tronco, utilizando-se de uma fita diamétrica com precisão de $\pm 1 \mathrm{~mm}$. Nas árvores que apresentavam raízes tabulares, o diâmetro foi medido acima da região de influência das raízes. A altura foi medida com uma trena e um clinômetro, usando o método do triângulo retângulo com ângulo de $45^{\circ}$. As espécies de árvores estranguladoras foram incluídas no censo também na forma de hemiepífita, quando pelo menos uma de suas raízes aéreas apresentava $\mathrm{DAP} \geq 10 \mathrm{~cm}$. As exsicatas foram depositadas nos herbários HUESC (Universidade Estadual de Santa Cruz, Ilhéus, BA) e CEPEC (Centro de Pesquisas do Cacau, Itabuna, BA). O segundo censo foi realizado após sete anos, em 2005,

Tabela 1. Propriedades químicas e granulometria do solo à profundidade de $0-20 \mathrm{~cm}$ em uma área de cabruca na região sul da Bahia.

\begin{tabular}{|c|c|c|c|c|c|c|c|}
\hline \multirow{2}{*}{$\begin{array}{l}\mathrm{pH} \\
\mathrm{H}_{2} 0\end{array}$} & $\mathrm{Al}$ & $\mathrm{Ca}$ & $\mathrm{Mg}$ & K & \multirow{2}{*}{$\frac{\mathrm{P}}{\mathrm{mg} / \mathrm{dm}^{3}}$} & $\mathrm{~N}$ & $\mathrm{C}$ \\
\hline & \multicolumn{4}{|c|}{$\mathrm{cmol}_{\mathrm{C}} / \mathrm{dm}^{3}$} & & \multicolumn{2}{|c|}{$\mathrm{g} / \mathrm{kg}$} \\
\hline 4,8 & 0,7 & 2,0 & 1,2 & 0,11 & 3 & 0,98 & 11,52 \\
\hline \multirow[t]{2}{*}{$\mathrm{Fe}$} & $\mathrm{Mn}$ & $\mathrm{Zn}$ & $\mathrm{Cu}$ & Areia grossa & Areia fina & Silte & Argila \\
\hline & \multicolumn{3}{|c|}{$\mathrm{mg} / \mathrm{dm}^{3}$} & \multicolumn{4}{|c|}{$\mathrm{dag} / \mathrm{kg}$} \\
\hline 53 & 45 & 2 & 5 & 17,4 & 33,1 & 33,8 & 15,7 \\
\hline
\end{tabular}


quando foram realizadas novas medições de diâmetro e observadas a mortalidade e o recrutamento de indivíduos. Informações mais detalhadas sobre o manejo aplicado na cabruca estudada durante o período entre as coletas foram obtidas através de conversas com os agricultores que trabalham na área.

Foram calculados para cada espécie os parâmetros fitossociológicos de densidade, frequiência, área basal e VI (valor de importância) (Curtis \& McIntosh 1950). A diversidade de espécies foi estimada através do índice de Shannon, utilizando logaritmo de base natural (Magurran 1988). Para estimar a riqueza total da cabruca estudada, foi utilizado o estimador desenvolvido por Chao \& Lee (1992), o qual se baseia no conceito estatístico de cobertura de amostragem, utilizando o número de espécies com baixa freqüência para estimar o número de espécies não incluídos na amostragem e, assim, o número total de espécies da área (Colwell \& Coddington 1994; Chazdon et al. 1998). Os cálculos foram feitos através do programa EstimateS, versão 6.0 (Colwell 2000). O mesmo programa foi utilizado para fazer a curva de aumento do número de espécies com o aumento da área amostrada (curva espécie-área), utilizando os valores médios obtidos em 1.000 combinações aleatórias de parcelas. Para comparar a composição florística da área estudada com a de outras áreas de cabruca levantadas na região (Sambuichi 2002; R.H.R. Sambuichi, dados não publicados) foi utilizado o índice de Sørensen qualitativo (Mueller-Dumbois \& Ellenberg 1974). As taxas de mortalidade e recrutamento foram obtidas pela fórmula algébrica de acordo com Sheil et al. (1995).

\section{Resultados e discussão}

Fitossociologia da área (censo de 1998) - Foram encontrados na área 120 indivíduos pertencentes a 62 espécies. Quatro indivíduos encontravam-se na forma de hemiepífita, pertencentes às espécies de árvores estranguladoras Ficus clusiaefolia (2), Ficus trigona (1) e Ficus broadwayi (1). Os maiores VIs foram apresentados por Sterculia excelsa, Sloanea obtusifolia e Guettarda platyphylla (Tab. 2), espécies características de florestas maduras. Todas as espécies encontradas na área estudada são nativas, quase todas ocorrendo naturalmente nas florestas mais conservadas da região, com exceção das espécies heliófitas de ciclo curto Trema micrantha e Albizia polycephala, e de algumas espécies estranguladoras, como Ficus clusiaefolia, as quais são características de áreas abertas e perturbadas. Esses dados diferem do encontrado em levantamentos feitos em áreas de cabruca antiga, onde a vegetação arbórea foi dominada por uma ou duas espécies de árvores frutíferas exóticas (M. Hummel, dados não publicados; Sambuichi 2002). Diferem também dos resultados de outros levantamentos realizados em áreas de cabruca nova (R.H.R. Sambuichi, dados não publicados), onde as espécies de maior importância foram pioneiras ou secundárias iniciais, o que foi atribuído ao estado de abandono dos tratos culturais nessas áreas. Em todos esses levantamentos, porém, a densidade de indivíduos, área basal e o número de espécies de florestas maduras foi superior ao das espécies características dos estágios iniciais de sucessão e exóticas. Resultados diferentes foram encontrados por Rolim \& Chiarello (2004), trabalhando em cabrucas antigas no Espírito Santo, onde $56,2 \%$ das espécies, $71,0 \%$ do número de indivíduos e $72,3 \%$ da área basal total estavam relacionados a espécies pioneiras e secundárias iniciais.

Comparando os valores de densidade e área basal por hectare obtidos no presente trabalho e em diferentes levantamentos realizados em áreas de cabruca e floresta da região, é possível avaliar a intensidade do raleamento da vegetação arbórea das cabrucas (Tab. 3). Observa-se que os valores de densidade são muito variáveis. As variações observadas entre áreas de floresta podem estar relacionadas a diferenças no estado de perturbação das áreas e a condições de solo e relevo particulares. A densidade de indivíduos nas cabrucas depende da escolha pessoal do agricultor quanto à quantidade de sombra a ser deixada na plantação e também do tamanho dos indivíduos, pois árvores maiores produzem mais sombra por indivíduo. A recomendação da CEPLAC (principal órgão de fomento agrícola da região) é de deixar 25 árvores por hectare para aumentar a produtividade do cacau, mas, por razões econômicas relacionadas ao aumento da necessidade de insumos em áreas muito abertas, os agricultores raramente seguem essa recomendação (Johns 1999). A redução de área basal de árvores das florestas para as cabrucas é menor que a redução de densidade, o que já seria esperado, pois, durante o raleamento, são conservadas nas cabrucas as árvores de maior porte. Apesar do intenso raleamento das árvores, as cabrucas apresentam em geral fisionomia de vegetação fechada, sendo facilmente confundidas com as florestas à distância, devido à presença dos cacaueiros, que apresentam em média cerca de 700 ind. ha-1 (Gramacho et al. 1992).

A altura dos indivíduos na cabruca estudada variou de 9,1 a 47,8 m, com média de 30,0 m. O DAP variou 
Tabela 2. Número de indivíduos, área basal e VI de espécies arbóreas utilizadas para sombreamento de cacau em uma área de 1,7 ha de cabruca na região sul da Bahia.

\begin{tabular}{|c|c|c|c|c|c|c|}
\hline \multirow[b]{2}{*}{ Espécies } & \multicolumn{3}{|c|}{1998} & \multicolumn{3}{|c|}{2005} \\
\hline & $\begin{array}{l}\text { Número de } \\
\text { indivíduos }\end{array}$ & Área Basal & VI & $\begin{array}{l}\text { Número de } \\
\text { indivíduos }\end{array}$ & Área Basal & VI \\
\hline
\end{tabular}

Sterculia excelsa Mart.

Sloanea obtusifolia (Moric.) K. Schum.

Guettarda platyphylla Müll. Arg.

Pterocarpus rohrii Vahl

Eschweilera ovata (Cambess.) Miers

Ficus gomelleira Kunth \& C.D. Bouché

Cedrela odorata L.

Ficus trigona L. f.

Eriotheca sp.

Dialium guianense (Aubl.) Sandw.

Ocotea percurrens Vincentini

Ficus insipida Willd.

Hieronyma alchorneoides Allemao

Copaifera lucens Dwyer

Caryocar edule Casar.

Virola officinalis Warb.

Zanthoxylum minutiflorum Tul.

Nectandra sp. 1

Annonaceae A

Brosimum sp.

Simira glaziovii (K. Schum.) Steyerm.

Moldenhawera blanchetiana Tul.

Chrysophyllum splendens Spreng.

Lecythis pisonis Cambess.

Psidium sp.

Brosimum guianense (Aubl.) Huber

Macrolobium latifolium Vogel.

Albizia polycephala (Benth.) Killip

Lecythis lurida (Miers) S.A. Mori.

Ficus clusiaefolia Schott

Myrtaceae A

Luehea divaricata Mart.

Cryptocarya mandioccana Meisn.

Ficus pulchella Schott ex Spreng.

Licania sp. 1

Ballizia pedicellaris (DC.) Barneby \& J.W. Grimes

Myrtaceae B

Micropholis crassipedicellata Pierre

Copaifera trapezifolia Hayne

Schefflera morototoni (Aubl.) Maguirre, Steyerm \& Frodin.

Licaria bahiana $\mathrm{H}$. Kurz.

Rinorea guianensis Aubl.

Protium heptaphyllum (Aubl.) March.

Sloanea guianensis (Aubl.) Benth.

Rollinia sp.

Tabebuia elliptica (A. DC.) Sandw.

Apeiba albiflora Ducke

Myrtaceae C

Maytenus sp.

Licania discolor Pilg.

Nectandra sp. 2

Helicostylis tomentosa (Poepp. \& Endl.) Macbride

Myrtaceae D

Licania sp. 2

12

4
6

4

6

2

4

2

2

3

3

2

3

3

1

3
2

2

2

2

2

2

2

2

2

2

2

2

2

2

1

1

1

1

1
1

1

\section{1}

1
1

1
1

1
1

1
1

1
1
1

1

1

1

1

1

1
1

1
1

\begin{tabular}{|c|c|c|}
\hline 12 & 5,13 & 30,95 \\
\hline 4 & 2,59 & 13,44 \\
\hline 6 & 1,10 & 13,03 \\
\hline 4 & 1,93 & 11,76 \\
\hline 6 & 0,70 & 11,14 \\
\hline 2 & 1,92 & 8,31 \\
\hline 4 & 0,58 & 8,28 \\
\hline 2 & 1,66 & 7,66 \\
\hline 2 & 1,49 & 7,21 \\
\hline 3 & 0,81 & 7,19 \\
\hline 3 & 0,75 & 7,03 \\
\hline 2 & 1,35 & 6,87 \\
\hline 3 & 0,69 & 6,86 \\
\hline 3 & 0,59 & 6,61 \\
\hline 1 & 1,83 & 6,40 \\
\hline 3 & 0,75 & 6,17 \\
\hline 2 & 0,81 & 5,49 \\
\hline 2 & 0,64 & 5,04 \\
\hline 2 & 0,61 & 4,95 \\
\hline 2 & 0,51 & 4,72 \\
\hline 2 & 0,51 & 4,70 \\
\hline 2 & 0,44 & 4,54 \\
\hline 2 & 0,43 & 4,50 \\
\hline 2 & 0,41 & 4,46 \\
\hline 2 & 0,34 & 4,28 \\
\hline 2 & 0,32 & 4,23 \\
\hline 2 & 0,32 & 4,23 \\
\hline 2 & 0,26 & 4,07 \\
\hline 2 & 0,15 & 3,80 \\
\hline 2 & 0,10 & 3,66 \\
\hline 1 & 0,76 & 3,63 \\
\hline 1 & 0,66 & 3,40 \\
\hline 1 & 0,59 & 3,22 \\
\hline 1 & 0,57 & 3,17 \\
\hline 1 & 0,56 & 3,14 \\
\hline 1 & 0,56 & 3,13 \\
\hline 1 & 0,50 & 2,98 \\
\hline 1 & 0,44 & 2,84 \\
\hline 1 & 0,40 & 2,73 \\
\hline 1 & 0,39 & 2,70 \\
\hline 1 & 0,36 & 2,62 \\
\hline 1 & 0,36 & 2,62 \\
\hline 1 & 0,32 & 2,52 \\
\hline 1 & 0,29 & 2,44 \\
\hline 1 & 0,28 & 2,41 \\
\hline 1 & 0,26 & 2,37 \\
\hline 1 & 0,25 & 2,34 \\
\hline 1 & 0,24 & 2,33 \\
\hline 1 & 0,21 & 2,24 \\
\hline 1 & 0,18 & 2,17 \\
\hline 1 & 0,15 & 2,10 \\
\hline 1 & 0,15 & 2,08 \\
\hline 1 & 0,15 & 2,07 \\
\hline 1 & 0,14 & 2,07 \\
\hline
\end{tabular}

$\begin{array}{lrr}6 & 2,83 & 17,33 \\ 3 & 2,17 & 11,06 \\ 6 & 1,16 & 14,02 \\ 4 & 2,22 & 13,02 \\ 6 & 0,84 & 12,27 \\ 2 & 2,40 & 9,79 \\ 4 & 0,88 & 9,62\end{array}$

7,33
1,06
4,02
3,02
2,27
9,79
9,62

$2,06 \quad 8,94$

$0,72 \quad 3,69$

$0,89 \quad 7,79$

$0,96 \quad 7,97$

$1,47 \quad 7,44$

$0,85 \quad 7,70$

$0,63 \quad 7,13$

$1,84 \quad 6,52$

$0,67 \quad 5,39$

$0,89 \quad 5,96$

$0,79 \quad 5,71$

$0,63 \quad 5,29$

$0,27 \quad 2,52$

$0,57 \quad 5,13$

$0,45 \quad 4,83$

$0,16 \quad 4,10$

$0,47 \quad 4,89$

$0,40 \quad 4,69$

$0,36 \quad 4,59$

$0,34 \quad 4,54$

$0,13 \quad 2,17$

$0,15 \quad 4,07$

$1,73 \quad 8,10$

$0,79 \quad 3,84$

$0,62 \quad 3,42$

$0,57 \quad 3,30$

$0,62 \quad 3,43$

$0,57 \quad 3,29$

$0,61 \quad 3,39$

$0,56 \quad 3,27$

$0,43 \quad 2,94$

$0,48 \quad 3,08$

$0,55 \quad 3,23$

$0,37 \quad 2,80$

$0,43 \quad 2,94$

$0,38 \quad 2,82$

0,30

2,60

$0,26 \quad 2,52$

$0,26 \quad 2,52$

$-$

$0,18 \quad 2,30$

$0,15 \quad 2,22$

$0,17 \quad 2,27$

$0,15 \quad 2,23$ 
Tabela 2 (continuação)

\begin{tabular}{|c|c|c|c|c|c|c|}
\hline \multirow[b]{2}{*}{ Espécies } & \multicolumn{3}{|c|}{1998} & \multicolumn{3}{|c|}{2005} \\
\hline & $\begin{array}{l}\text { Número de } \\
\text { indivíduos }\end{array}$ & Área Basal & VI & $\begin{array}{l}\text { Número de } \\
\text { indivíduos }\end{array}$ & Área Basal & VI \\
\hline Drypetes sessiliflora Allemao & 1 & 0,13 & 2,04 & 1 & 0,17 & 2,27 \\
\hline Alseis floribunda Schott & 1 & 0,12 & 2,01 & 1 & 0,13 & 2,18 \\
\hline Tabebuia cassinoides (Lam.) DC. & 1 & 0,11 & 1,98 & 1 & 0,11 & 2,13 \\
\hline Sloanea monosperma Vell. & 1 & 0,08 & 1,91 & 1 & 0,15 & 2,21 \\
\hline Myrtaceae E & 1 & 0,08 & 1,90 & 1 & 0,09 & 2,06 \\
\hline Trema micrantha (L.) Blume & 1 & 0,03 & 1,79 & 5 & 0,19 & 8,75 \\
\hline Ficus broadwayi Urb. & 1 & 0,02 & 1,75 & 2 & 0,05 & 3,83 \\
\hline Talisia elephantipes Sandw. & 1 & 0,01 & 1,74 & - & - & - \\
\hline Cecropia sp. & - & - & - & 1 & 0,03 & 1,92 \\
\hline Total & 120 & 39,09 & 300,00 & 110 & 39,28 & 300,00 \\
\hline
\end{tabular}

de 13,1 a $153,0 \mathrm{~cm}$, sendo que $60 \%$ dos indivíduos apresentou DAP $\geq 50 \mathrm{~cm}$. A maior altura foi apresentada por um indivíduo da espécie Sterculia excelsa e o maior diâmetro pela espécie Caryocar edule (Tab. 4). A altura média foi maior do que a média encontrada em outras áreas de cabruca e floresta da região (Tab. 3). O DAP máximo encontrado foi muito maior que o de $89,2 \mathrm{~cm}$ encontrado por Mori et al. (1983) em uma floresta nativa. A densidade de indivíduos de grande porte (DAP $\geq 70 \mathrm{~cm}$ ) foi 19,4 ind. ha ${ }^{-1}$, valor alto quando comparado com as densidades de 8,9 e 10,6 ind. ha ${ }^{-1}$ observadas para áreas de floresta não perturbada no Panamá e na Costa Rica (Clark \& Clark 1996). Essa alta densidade de indivíduos de grande porte pode ser um reflexo do que existia na floresta original da cabruca estudada ou ser decorrente de um crescimento excepcional das árvores remanescentes na área. A distribuição de indivíduos em classes de altura e diâmetro (Fig. 1 A-B) mostrou grande predominância de indivíduos maduros, indicando haver muito pouco recrutamento de indivíduos jovens na área. Esse é um padrão observado em outras cabrucas estudadas (H. Hummel, dados não publicados; Sambuichi 2002), com exceção daquelas em que as práticas de manejo foram abandonadas (R.H.R. Sambuichi, dados não publicados). Isso ocorre porque a prática de manejo conhecida como roçagem, a qual consiste no corte periódico da vegetação rasteira, elimina a maioria das plântulas das árvores, permitindo o estabelecimento de apenas alguns indivíduos selecionados para cobrir falhas no sombreamento causadas pela morte das árvores originais (Sambuichi 2002).

O índice de diversidade de Shannon foi 3,88 nats ind. ${ }^{-1}$ (Tab. 5), valor comparável a áreas ricas de floresta tropical. Mais da metade das espécies (32) ocorreu com apenas um indivíduo na área estudada, havendo uma alta relação espécies/indivíduos $(0,52)$.

Tabela 3. Densidade, área basal e altura média de árvores* em levantamentos fitossociológicos realizados em áreas de cabruca e de floresta ombrófila densa na região sul da Bahia.

\begin{tabular}{|c|c|c|c|}
\hline & $\begin{array}{l}\text { Densidade } \\
\text { (ind. ha }{ }^{-1} \text { ) }\end{array}$ & $\begin{array}{c}\text { Área Basal } \\
\left(\mathrm{m}^{2} \mathrm{ha}^{-1}\right)\end{array}$ & $\begin{array}{c}\text { Altura } \\
(\mathrm{m})\end{array}$ \\
\hline \multicolumn{4}{|l|}{ Cabrucas } \\
\hline Presente trabalho & 70,5 & 23,0 & 30,0 \\
\hline Sambuichi (2002) (uma área) & 53,1 & 20,0 & 15,9 \\
\hline M. Hummel (não publicado) (duas áreas) & 48,8 e 54,4 & - & 19,5 \\
\hline M.C. Alves (não publicado) (quatro áreas) & 35,0 a 132,9 & - & 16,4 a 21,4 \\
\hline R.H.R. Sambuichi (não publicado) (cinco áreas) & 47,3 a 355,0 & 11,8 a 28,2 & \\
\hline \multicolumn{4}{|l|}{ Florestas } \\
\hline Mori et al. 1983 (uma área) & 891,3 & 46,3 & - \\
\hline M.C. Alves (não publicado) (quatro áreas) & 596,0 a 829,6 & - & - \\
\hline W.W. Thomas et al. (não publicado) (uma área) & 988,0 & 39,6 & 17,8 \\
\hline
\end{tabular}

*DAP (diâmetro à altura do peito) $\geq 10 \mathrm{~cm}$. 
Tabela 4. Espécie, altura e diâmetro* dos indivíduos arbóreos com mais de $35 \mathrm{~m}$ de altura encontrados em uma área de 1,7 ha de cabruca na região sul da Bahia. Medições realizadas nos anos de 1998 e 2005.

\begin{tabular}{|c|c|c|c|}
\hline \multirow[t]{2}{*}{ Espécie } & \multicolumn{2}{|c|}{1998} & \multirow{2}{*}{$\begin{array}{c}2005 \\
\begin{array}{c}\text { Diâmetro } \\
(\mathrm{cm})\end{array}\end{array}$} \\
\hline & $\begin{array}{l}\text { Altura } \\
(\mathrm{m})\end{array}$ & $\begin{array}{l}\text { Diâmetro } \\
\quad(\mathrm{cm})\end{array}$ & \\
\hline Sterculia excelsa Mart. & 47,8 & 98,7 & morta \\
\hline Sloanea obtusifolia (Moric.) K. Schum. & 46,4 & 100,3 & 105,1 \\
\hline Caryocar edule Casar. & 43,1 & 152,9 & 153,0 \\
\hline Micropholis crassipedicellata Pierre & 42,9 & 75,2 & 84,4 \\
\hline Sterculia excelsa Mart. & 42,6 & 70,1 & morta \\
\hline Licaria bahiana H. Kurz. & 41,6 & 67,5 & 83,4 \\
\hline Sterculia excelsa Mart. & 41,2 & 65,6 & morta \\
\hline Pterocarpus rohrii Vahl & 39,8 & 113,7 & 115,0 \\
\hline Ficus gomelleira Kunth \& C.D. Bouché ** & 39,6 & 149,7 & 160,2 \\
\hline Ficus trigona L.f. $* *$ & 37,7 & 126,8 & 141,0 \\
\hline Eriotheca sp. & 37,7 & 111,5 & morta \\
\hline
\end{tabular}

*Diâmetro medido à altura de 1,30 m do solo (DAP) ou acima da área de influência das raízes. **Para essas espécies estranguladoras não foi possível medir o diâmetro acima da área de influênciadas raízes, sendo medido o diâmetro total das raízes a $1,30 \mathrm{~m}$ do solo.

A riqueza total estimada (Chao) foi de 110 espécies (Tab. 5). Altos índices de diversidade, variando entre 3,31 a 4,22 nats ind..$^{-1}$, foram encontrados em áreas de cabruca (H. Hummel, dados não publicados; Sambuichi 2002; R.H.R. Sambuichi, dados não publicados), o que reflete em parte a alta diversidade natural das florestas da região (Mori et al. 1983). O índice de similaridade florística (Sørensen) entre a área estudada e outras áreas de cabruca levantadas no mesmo município (Sambuichi 2002; R.H.R. Sambuichi, dados não publicados), variou entre 0,25 e 0,43 com média de 0,29, mostrando haver baixa similaridade entre áreas. Baixas similaridades florísticas são comuns entre áreas adjacentes de floresta pluvial tropical (Campbell et al. 1986) e, no caso das cabrucas, pode ser um reflexo do que já existia na floresta original. As cabrucas antigas, em geral, apresentam maiores índices de similaridade entre si, o que decorre principalmente da dominância de espécies exóticas nessas áreas (R.H.R. Sambuichi, dados não publicados). Sendo as cabrucas áreas cultivadas, a similaridade entre elas é também influenciada por diferenças nas práticas de manejo e no estado sucessional das áreas. Os altos índices de diversidade observados em cada área e a baixa similaridade entre áreas próximas mostram que as cabrucas apresentam elevada diversidade alfa e beta de espécies arbóreas, indicando, conseqüentemente, que uma significativa diversidade gama (regional) de árvores encontra-se ainda nessas áreas de cultivo, apesar da influência antrópica.

Dinâmica da vegetação (período de 1998-2005) - No segundo censo, após sete anos, foram encontrados na área 110 indivíduos pertencentes a 58 espécies, sendo três indivíduos na forma de hemiepífita. Morreram 17 indivíduos durante o período, pertencentes a 12 espécies diferentes, enquanto sete novos indivíduos foram recrutados, pertencentes a três espécies. A densidade foi de 64,7 ind. ha ${ }^{-1}$, havendo redução de $8,4 \%$ no período. Sterculia excelsa continuou sendo a espécie de maior VI na área (Tab. 2), mas apresentou uma substancial redução de importância (44\%) e densidade (50\%). Essa é uma espécie de grande porte e madeira pouco resistente, a qual não apresenta raízes superficiais para sustentação, como raízes tabulares ou escoras, sendo por isso muito suscetível a ser derrubada ou quebrada pelo vento. Três das árvores mortas dessa espécie estavam entre os indivíduos mais altos encontrados na área (Tab. 4).

A taxa de mortalidade para o total das espécies foi de $2,16 \%$ ano $^{-1}$ e a taxa de recrutamento foi de $0,81 \%$ ano $^{-1}$. As taxas obtidas estão dentro da faixa de valores obtidos para florestas tropicais. Phillips \& Gentry (1994) revisando dados de 22 levantamentos realizados em áreas de floresta tropical em períodos mais recentes, encontraram valores de taxa de mortalidade variando de 0,51 a $3,63 \%$ ano $^{-1}$, com média de 2,00\% ano $^{-1}$. Gomes et al. (2003), compilando dados de oito levantamentos feitos em parcelas permanentes em áreas de floresta tropical, encontrou valores de taxa de mortalidade entre 0,67 e $2,64 \%$ ano $^{-1}$ e taxas de recrutamento entre 0,40 e 3,46\% ano ${ }^{-1}$. Lewis et al. (2004) mostraram, porém, que as taxas anuais de 


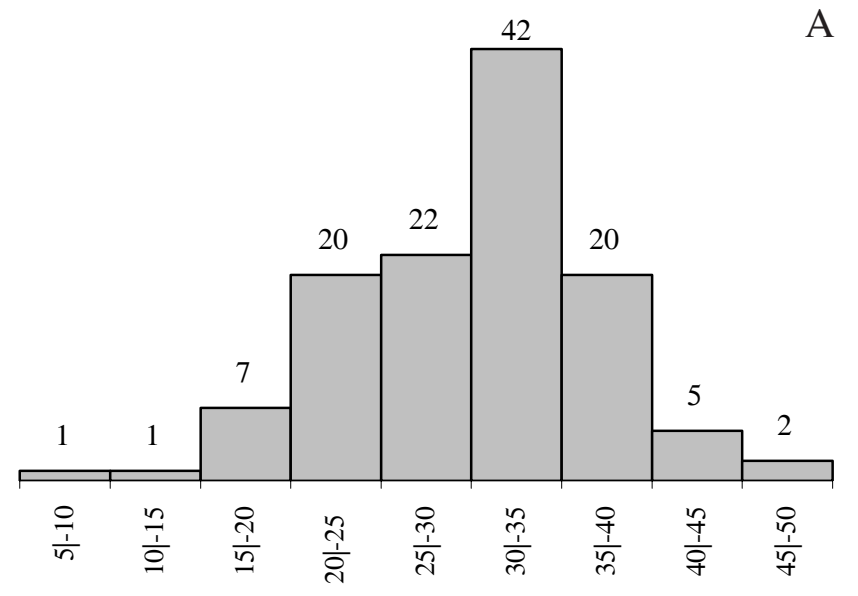

Classe de altura $(\mathrm{m})$

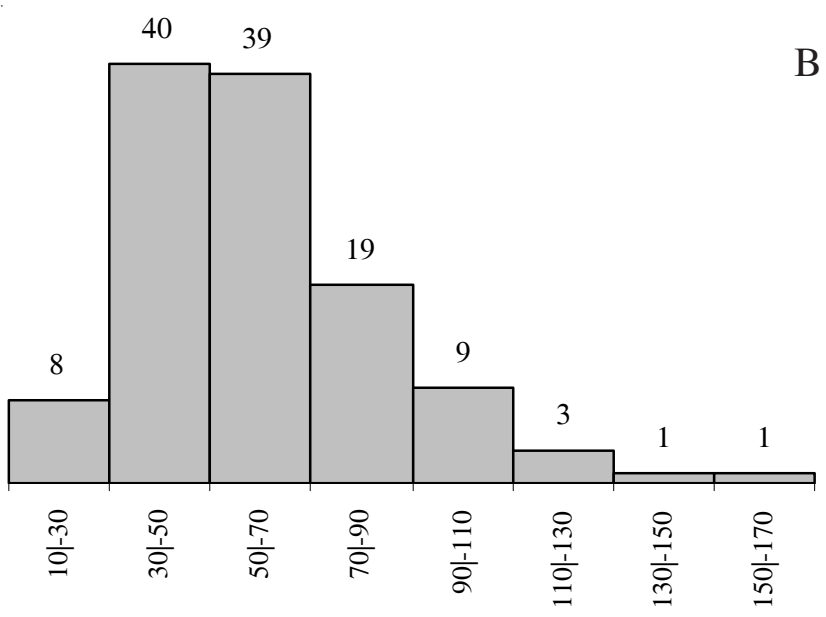

Classe de diâmetro $(\mathrm{cm})$

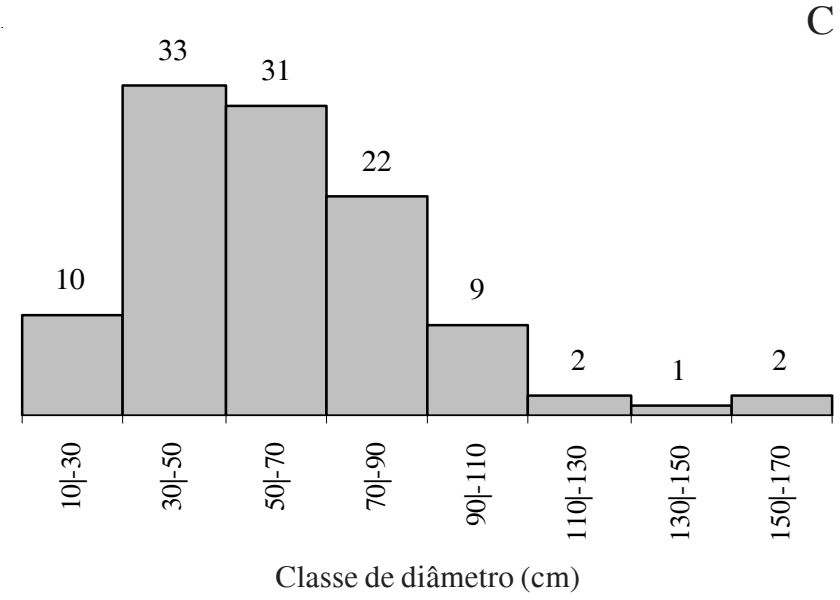

Figura 1. Distribuição de tamanho para árvores sombreadoras de cacau em uma área de 1,7 ha de cabruca na região sul da Bahia. A. Classe de altura, dados de 1998. B. Classe de diâmetro, dados de 1998. C. Classe de diâmetro, dados de 2005. Diâmetro medido à altura de 1,30 m do solo (DAP) ou acima da área de influência das raízes.
Tabela 5. Diversidade, eqüitatividade e riqueza de espécies para árvores encontradas nos anos de 1998 e 2005 em uma área de 1,7 ha de cabruca na região sul da Bahia.

\begin{tabular}{lcc}
\hline Espécie & 1998 & 2005 \\
\hline $\begin{array}{l}\text { Diversidade de espécies } \\
\quad \text { Shannon } H^{\prime} \text { (nats/ind.) }\end{array}$ & 3,88 & 3,87 \\
$\begin{array}{l}\text { Eqüitatividade (evenness) } \\
\quad \text { Pielou J (nats/ind.) }\end{array}$ & 0,94 & 0,95 \\
$\begin{array}{l}\text { Riqueza de espécies } \\
\quad \text { Número de espécies encontradas }\end{array}$ & 62 & 58 \\
$\quad$ Riqueza total estimada (Chao) & 110 & 90 \\
\hline
\end{tabular}

mortalidade e recrutamento calculadas para todas as espécies em conjunto além de variarem com as condições particulares de cada floresta, como solo, clima, composição de espécies e perturbações, variam também com as condições de amostragem. O intervalo de tempo entre as coletas de dados e o tamanho da amostra exercem influência sobre as taxas obtidas em cada levantamento, o que dificulta em muitos casos as comparações. No caso da área estudada, observa-se que a diferença entre a taxa de mortalidade e a taxa recrutamento foi alta, mostrando existir um forte balanço negativo, o qual resultou na redução de densidade observada entre o primeiro e o segundo censo.

Apesar da redução de densidade, a área basal total aumentou no período devido ao crescimento dos indivíduos. A taxa absoluta média de incremento diamétrico para os indivíduos vivos foi de $0,7 \pm$ $0,6 \mathrm{~cm} \mathrm{ano}^{-1}$, variando de $-0,1 \mathrm{a} 2,6 \mathrm{~cm} \mathrm{ano}^{-1}$. O menor incremento foi apresentado por um indivíduo da espécie Lecythis lurida e o maior pela espécie Ficus gomelleira. Esses resultados mostraram existir na área elevada taxa de incremento diamétrico médio em comparação a áreas de floresta tropical natural. Silva et al. (2002), trabalhando na Amazônia central, encontraram taxa média de incremento de 0,16 \pm $0,02 \mathrm{~cm}$ ano $^{-1}$, com variação entre indivíduos de $-0,05$ a $1,14 \mathrm{~cm} \mathrm{ano}^{-1}$. Veira et al. (2004), também na Amazônia, encontraram taxas médias de incremento de 0,39 $\mathrm{cm}^{\text {ano }}{ }^{-1}$ em Rio Branco, 0,31 $\mathrm{cm}^{2} o^{-1} \mathrm{em}$ Santarém e $0,17 \mathrm{~cm}^{2} \mathrm{ano}^{-1}$ em Manaus.

Vieira et al. (2004) discutiram alguns fatores que podem ser responsáveis por diferenças entre as taxas de crescimento observadas entre áreas de floresta, como: condições climáticas, disponibilidade de radiação luminosa, propriedades do solo, disponibilidade de água, composição de espécies e perturbações. No caso das 
cabrucas, o raleamento das árvores diminui a competição por uso de recursos, aumentando a disponibilidade de luz, nutrientes e água para os indivíduos. Mesmo incluindo os cacaueiros, a densidade de indivíduos ainda é em geral menor que a observada em florestas. Além disso, os cacaueiros são árvores pequenas, de forma que as árvores sombreadoras, mais altas e bem espaçadas, encontram na cabruca uma alta disponibilidade de radiação luminosa, podendo assim se desenvolver mais rapidamente e atingir grande porte. Outro fator que pode também contribuir para o maior desenvolvimento das árvores é o aumento da disponibilidade de nutrientes através de adubação e calagem artificiais feitos periodicamente nessas áreas para estimular a produtividade da cultura. Estudos realizados em áreas de floresta mostraram que as taxas de incremento diamétrico variam significativamente com a classe de diâmetro (Clark \& Clark 1999) e são em geral maiores para os indivíduos de maior diâmetro (Silva et al. 2002; Vieira et al. 2004). Explica-se que os indivíduos de grande porte, já estando bem estabelecidos na área, conseguem ter acesso a maior quantidade de recursos que os menores. Na cabruca estudada não foi observada diferença significativa de crescimento entre as classes de diâmetro (ANOVA, $\mathrm{P}>0,10)$, indicando que o raleamento otimiza a obtenção de recursos para árvores sombreadoras de todos os tamanhos (Tab. 6).

A proporção de indivíduos mortos foi maior entre as árvores com mais de $30 \mathrm{~m}$ de altura e entre as com mais de $50 \mathrm{~cm}$ de diâmetro. Enquanto as classes de diâmetro de 30 a $50 \mathrm{~cm}$ e 50 a $70 \mathrm{~cm}$ apresentaram redução em número e indivíduos, as classes de 10 a $30 \mathrm{~cm}$ e 70 a $90 \mathrm{~cm}$ aumentaram devido ao recrutamento de indivíduos vindo de classes de tamanho anteriores (Fig. 1 B-C). O número de indivíduos de grande porte (DAP $\geq 70 \mathrm{~cm}$ ) aumentou de 33 para 36 no período, mostrando que, para estes, a

Tabela 6. Taxas médias anuais de incremento diamétrico por classe de diâmetro para árvores sombreadoras de cacau em uma área de 1,7 ha de cabruca na região sul da Bahia, no período de 1998 a 2005.

\begin{tabular}{ccc}
\hline \multirow{2}{*}{ Classes de diâmetro $(\mathrm{cm})$} & \multicolumn{2}{c}{ Incremento $\left(\mathrm{cm} \mathrm{ano}^{-1}\right)$} \\
\cline { 2 - 3 } & Médio & Mínimo e máximo \\
\hline $10 \mid-30$ & $1,0 \pm 0,8$ & $(0,0-2,5)$ \\
$30 \mid-50$ & $0,6 \pm 0,6$ & $(-0,1-2,6)$ \\
$50 \mid-70$ & $0,8 \pm 0,6$ & $(0,0-2,3)$ \\
$\geq 70$ & $0,7 \pm 0,6$ & $(0,0-2,2)$ \\
\hline
\end{tabular}

perda por mortalidade foi compensada pelo crescimento dos indivíduos. A diferença entre a distribuição indivíduos por classe de diâmetro no primeiro e no segundo censo, porém, não foi considerada significativa (Kolmogorov-Smirnov, $\mathrm{D}_{\max }=0,1368<\mathrm{D}_{00,5}=0,3524$ ).

A riqueza de espécies por área amostrada diminuiu durante o período entre coletas (Fig. 2). Cinco espécies desapareceram (Licania discolor, Luehea divaricata, Maytenus sp., Rollinia sp. e Talisia elephantipes) e apenas uma nova espécie foi adicionada (Cecropia sp.). A redução no índice de diversidade, porém, foi pequena (Tab. 5), não sendo considerada significativa $(t=0,058, P>0,10)$, pois embora a riqueza tenha diminuído houve aumento de eqüitatividade (evenness) devido à redução de densidade da espécie dominante Sterculia excelsa. As estimativas de riqueza total (Chao) (Tab. 5) indicaram redução de cerca de vinte espécies na cabruca estudada durante o período, o que daria uma taxa média de quase três espécies perdidas por ano. Esses dados chegam a ser alarmantes, pois indicam haver acelerado empobrecimento da vegetação na área de estudo. Além disso, dos sete novos indivíduos recrutados no período, cinco pertenceram à espécie pioneira Trema micrantha, um à espécie pioneira Cecropia sp. e um, na forma de hemiepífita, à espécie estranguladora Ficus broadwayi, mostrando que, embora vários indivíduos de espécies de florestas maduras tenham morrido, apenas indivíduos de espécies heliófitas de crescimento rápido, características de áreas perturbadas, foram recrutados.

Informações fornecidas pelos trabalhadores rurais ajudam a explicar os resultados obtidos. Segundo os

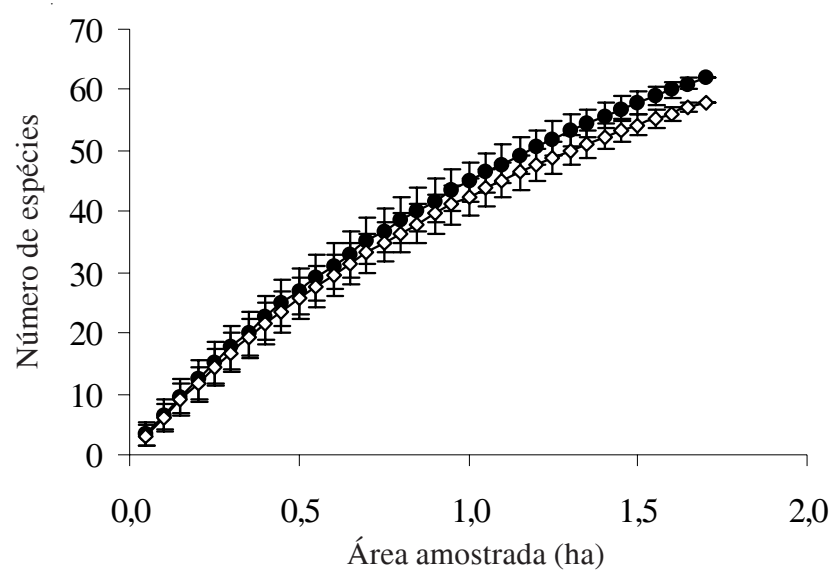

Figura 2. Curva "espécie-área” para árvores sombreadoras de cacau em uma área de 1,7 ha de cabruca na região sul da Bahia. Dados coletados nos anos de $1998(-\bullet-)$ e $2005(-\diamond-)$. 
mesmos, o dono da área considera que existe ainda um excesso de sombreamento na cabruca estudada e por essa razão os trabalhadores são orientados a não permitir o recrutamento de novas árvores, cortando todas as plântulas durante a roçagem. Os poucos indivíduos recrutados durante o período de estudo escaparam do corte devido ao seu crescimento rápido, mas serão certamente eliminados em roçagens posteriores, pois os agricultores em geral não gostam de manter essas espécies pioneiras de pequeno porte e madeira sem utilidade nas plantações. Esse processo de raleamento provavelmente continuará na área até que se considere que já existe necessidade de repor as árvores mortas.

Dois problemas principais podem então ser identificados em relação à conservação de espécies arbóreas nativas nas cabrucas. Primeiro, as recomendações agronômicas de manejo das cabrucas visam apenas à produtividade de cacau das áreas, sem levar em conta o valor das árvores para conservação. Milhões de árvores foram derrubadas nas cabrucas no passado devido às recomendações de raleamento de sombra (Alvim 1966) e no presente esse raleamento ainda continua através de corte clandestino ou, como no presente caso, devido à inibição do recrutamento. Isso resulta em um significativo empobrecimento de espécies nas cabrucas, principalmente nas áreas mais novas, as quais são em geral mais densas e com maior proporção de espécies climáxcicas. R.H.R. Sambuichi (dados não publicados) mostrou, através de curvas de rarefação, que as cabrucas mais densas apresentam maior número de espécies por área e também por número de indivíduos amostrados, explicando que as áreas mais sombreadas apresentam maior proporção de espécies de floresta fechada, as quais apresentam maior riqueza natural do que as espécies pioneiras e secundárias iniciais. Outro problema, identificado anteriormente por Sambuichi (2002) e confirmado no presente trabalho, está relacionado à seleção para o recrutamento de novas árvores e leva à substituição de espécies observada nas cabrucas antigas. Mesmo nas áreas onde a cobertura de cacaueiros é mais densa e a baixa luminosidade no solo favorece a regeneração de espécies climáxcicas, os agricultores raramente reconhecem as plântulas dessas espécies por serem mais raras e sem utilidade conhecida. Quando eles consideram que existe a necessidade de reposição de sombra na plantação, geralmente selecionam plântulas das espécies mais conhecidas, de crescimento rápido e de valor econômico, podendo também recorrer à introdução de espécies exóticas.
Os resultados reforçaram a afirmação de que existe um importante banco de árvores nativas nas cabrucas, principalmente de espécies de grande porte, mostrando o grande potencial de uso dessas áreas para a conservação de espécies arbóreas. As medidas de dinâmica, porém, confirmaram as indicações obtidas em levantamentos fitossociológicos anteriores (Sambuichi 2002; Rolim \& Chiarello 2004) de que esse banco genético não está sendo adequadamente conservado e as cabrucas estão sendo rapidamente alteradas, raleadas e empobrecidas. Isso alerta para a necessidade urgente de modificar a forma de manejo dessas áreas de maneira a permitir a continuidade das espécies nativas de florestas maduras.

É preciso encontrar alternativas de compensação econômica aos produtores de cacau para incentivar que eles conservem as espécies nativas nas plantações. Devido à crise econômica causada pelos baixos preços do produto e a doenças na cultura, os produtores vêm procurando novas fontes complementares de renda e em muitos casos substituindo as árvores sombreadoras nativas por exóticas de interesse econômico. Os altos valores de área basal e as altas taxas de incremento diamétrico encontradas no presente trabalho dão um indicativo de que as cabrucas podem ser utilizadas para o seqüestro e estoque de carbono. É recomendável que novos estudos sejam realizados, utilizando metodologia mais precisa e acurada, além de amostragens mais abrangentes que incluam as variações de condições observadas nessas áreas e, também, o crescimento e a biomassa das árvores de cacau, para avaliar o balanço de carbono das cabrucas e o seu potencial de ganho econômico através do mercado de carbono.

\section{Agradecimentos}

Agradeço à Universidade Estadual de Santa Cruz, pelo apoio financeiro e material ao trabalho; a Jomar G. Jardim, do herbário da CEPLAC, pelo auxílio na coleta e identificação do material botânico; ao Sr. Henrique Cardoso, proprietário da área estudada, por permitir a pesquisa em sua fazenda; aos colegas Adriana M.Z. Martini, Marcelo S. Mielke e Leandro L. Loguercio, pelas valiosas sugestões à análise de dados e redação do trabalho.

\section{Referências bibliográficas}

Alvim, P.T. 1966. O problema do sombreamento do cacaueiro. Cacau Atualidades 3(2): 2-5. 
Araújo, M.; Alger, K.; Rocha, R. \& Mesquita, C.A.B. 1998. A Mata Atlântica do sul da Bahia: situação atual, ações e perspectivas. Reserva da Biosfera da mata Atlântica MAB - UNESCO, Caderno 8: 1-36.

Bawa, K.S. \& Ashton, P.S. 1991. Conservation of rare trees in tropical rain forests: a genetic perspective. Pp. 62-71. In: D.A. Falk \& K.E. Holsinger (eds.). Genetics and conservation of rare plants. Oxford, Oxford University Press.

Campbell, D.G.; Daly, D.C.; Prance, G.T. \& Maciel, U.N. 1986. Quantitative ecological inventory of terra firme and varzea tropical forest on Rio Xingu, Brazilian Amazon. Brittonia 38: 369-393.

Chao, A. \& Lee, S.M. 1992. Estimating the number of classes via sample coverage. Journal of the American Statistical Association 87: 210-217.

Chazdon, R.L.; Colwell, R.K.; Denslow, J.S. \& Guariguata, M.R. 1998. Statistical methods for estimating species richness of woody regeneration in primary and secondary rain forests of NE Costa Rica. Pp. 285-309. In: F. Dallmeier \& J.A. Comiskey (eds.). Forest biodiversity research, monitoring and modeling: conceptual background and Old World case studies. Paris, Parthenon Publishing.

Clark, D.B. \& Clark, D.A. 1996. Abundance, growth and mortality of very large trees in neotropical lowland rain forest. Forest Ecology and Management 80: 235-244.

Clark, D.B. \& Clark, D.A. 1999. Assessing the growth of tropical rainforest trees: issues for forest modeling and management. Ecological Applications 9(3): 981-997.

Colwell, R.K. 2000. EstimateS: Statistical estimation of species richness and shared species from samples. Version 6. User's Guide and Software published at: http:/ /viceroy.eeb.uconn.edu/estimates.

Colwell, R.K. \& Coddington, J.A. 1994. Estimating terrestrial biodiversity through extrapolation.Philosophical Transactions of the Royal Society (Series B) 345: 101-118.

Curtis, J.T. \& McIntosh, R.P. 1950. The interrelations of certain analytic and synthetic phytosociological characters. Ecology 31: 434-455.

Dietz, J.M.; Sousa, S.N. \& Billerbeck, R. 1996. Population dynamics of golden-headed lion tamarins Leontopithecus chysomelas in Una Reserve, Brazil. Dodo, Journal of the Jersey Wildlife Preservation Trust 32: 115-122.

Gallina, S.; Mandujano, S. \& Gonzalez-Romero A. 1996. Conservation of mammalian biodiversity in coffee plantations of Central Veracruz, Mexico. Agroforestry Systems 33: 13-27.

Gomes, E.P.C.; Mantovani, W. \& Kageyama P.Y. 2003. Mortality and recruitment of trees in a secondary rain forest in southeastern Brazil. Brazilian Journal of Biology 63(1): 47-60.

Gouvêa, J.B.S.; Silva, L.A.M. \& Hori, M. 1976. Fitogeografia. Pp.1-7. In: Diagnóstico socioeconômico da região cacaueira. v.7. Recursos florestais. Ilhéus, CEPLAC/ IICA.

Gramacho, I.C.P.; Magno, A.E.S.; Mandarino, E.P. \& Matos, A. 1992. Cultivo e beneficiamento do cacau na Bahia. Ilhéus, CEPLAC.
Greenberg, R.; Bichier, P. \& Sterling, J. 1997. Bird populations in rustic and planted shade coffee plantations of eastern Chiapas, Mexico. Biotropica 29(4): 501-514.

Johns, N.D. 1999. Conservation in Brazil's chocolate forest: the unlikely persistence of the traditional cocoa agroecosystem. Environmental Management 23(1): 31-47.

Johnson, M.D. 2000. Effects of shade-tree species and crop structure on the winter arthropod and bird communities in a Jamaican shade coffee plantation. Biotropica 32(1): 133-145.

Lewis, S.L.; Phillips, O.L.; Sheil, D.; Vinceti, B.; Baker, T.R.; Brown, S.; Graham, A.W.; Higuchi, N.; Hilbert, D.W.; Laurance, W.F.; Lejoly, J.; Malhi, Y.; Monteagudo, A.; Vargas P.N.; Sonké, B.; Supardi, N.; Terborgh, J.W. \& Martínez, R.V. 2004. Tropical forest tree mortality, recruitment and turnover rates: calculation, interpretation and comparison when census intervals vary. Journal of Ecology 92: 929-944.

Magurran, A.E. 1988. Ecological Diversity and its Measurements. London, Croom Helm.

Moguel, P. \& Toledo, V.M. 1999. Biodiversity conservation in tradicional coffee systems of Mexico. Conservation Biology 13(1): 11-21.

Mori, S.A.; Boom, B.M.; Carvalho, A.M. \& Santos, T.S. 1983. Southern bahian moist forests. Botanical Review 49(2): 155-232.

Mueller-Dumbois, D. \& Ellenberg, H. 1974. Aims and methods of vegetation ecology. New York, John Wiley.

Myers, N.; Mittermeier, R.A.; Mittermeier, C.G.; Fonseca, G.A.B. \& Kent, J. 2000. Biodiversity hotspots for conservation priorities. Nature 403: 853-858.

Nobre, S.S. 1998. The Atlantic rainforest in the Brazilian cocoa region. Applied Geography and Development 52: 41-51.

Pardini, R. 2004. Effects of forest fragmentation on small mammals in an Atlantic Forest landscape. Biodiversity and Conservation 13: 2567-2586.

Perfecto, I.; Rice, R.A.; Greenberg, R. \& Van der Voort, M.E. 1996. Shade coffee: a disappearing refuge for biodiversity. BioSience 46(8): 598-608.

Perfecto, I.; Vandermeer, J.; Hanson, P. \& Cartín, V. 1997. Arthropod biodiversity loss and the transformation of a tropical agro-ecosystem. Biodiversity and Conservation 6: $935-945$.

Perfecto, I.; Mas, A.; Dietsch, T. \& Vandermeer, J. 2003. Conservation of biodiversity in coffee agroecosystems: a tri-taxa comparison in southern Mexico. Biodiversity and Conservation 12: 1239-1252.

Phillips, O.L. \& Gentry, A.H. 1994. Increasing turnover through time in tropical forests. Science 263: 954-958.

Pimentel, D.; Stachow, U.; Takacs, D.A.; Brubaker, H.W.; Dumas A.R.; Meaney, J.J.; O’Neil, A.S.; Onsi, D.E. \& Corzilius, D.B. 1992. Conserving biological diversity in agricultural/ forestry systems. BioScience 42(5): 354-362.

Raboy, B.E.; Christman, M.C. \& Dietz, J.M. 2004. The use of degraded and shade cocoa forests by Endangered golden-headed lion tamarins Leontopithecus chrysomelas. Oryx 38(1): 75-83. 
Reitsma, R.; Parrish, J.D. \& McLarney, W. 2001. The role of cacao plantations in maintaining forest avian diversity in southeastern Costa Rica. Agroforestry Systems 53: 185-193.

Rice, R.A. \& Greenberg, R. 2000. Cacao Cultivation and the Conservation of Biological Diversity. Ambio 29(3): 167-173.

Rolim, S.G. \& Chiarello, A.G. 2004. Slow death of Atlantic forest trees in cocoa agroforestry in southeastern Brazil. Biodiversity and Conservation 13: 2679-2694.

Saatchi, S.; Agosti, D.; Alger, K.; Delabie, J. \& Musinsky, J. 2001. Examining fragmentation and loss of primary forest in the Southern Bahian Atlantic Forest of Brazil with radar imagery. Conservation Biology 15(4): 867-875.

Sambuichi, R.H.R. 2002. Fitossociologia e diversidade de espécies arbóreas em cabruca (mata Atlântica raleada sobre plantação de cacau) na região sul da Bahia, Brasil. Acta Botanica Brasilica 16(1): 89-101.

Schroth, G.; Fonseca G.A.B.; Harvey, C.A.; Gascon, C.; Vasconcelos H.L. \& Izac, A.M.N. (eds.). 2004. Agroforestry and Biodiversity Conservation in Tropical Landscapes. Washington, Island Press.

Sheil, D.; Burslem, D.F.R.P. \& Alder D. 1995. The interpretation and misinterpretation of mortality rate measures. Journal of Ecology 83: 331-333.
Silva, R.P.; Santos, J.; Tribuzy, E.S.; Chambers, J.Q.; Nakamura, S. \& Higuchi, N. 2002. Diameter increment and growth patterns for individual tree growing in Central Amazon, Brazil. Forest Ecology and Management 166: 295-301.

Sperber, C.F.S.; Nakayama, K.; Valverde, M.J. \& Neves, F.S. 2004. Tree species richness and density affect parasitoid diversity in cacao agroforestry. Basic and Applied Ecology 5: 241-251.

Thomas, W.W. \& Carvalho, A.M. 1997. Atlantic moist forest of southern Bahia. Pp. 364-368. In: S.D. Davis; V.H. Heywood; O.H. MacBryde \& A.C. Hamilton (eds.). Centres of plant diversity: a guide and strategy for their conservation. v.3, London, IUCN-WWF.

Thomas, W.W.; Carvalho, A.M.; Amorim, A.M.A.; Garrison, J. \& Arbeláez, A.L. 1998. Plant endemism in two forests in southern Bahia, Brasil. Biodiversity and Conservation 7: 311-322.

Vieira, S.; Camargo, P.B.; Selhorst, D.; Silva, R.; Hutyra, L.; Chambers, J.Q.; Brown, I.F.; Higuchi, N.; Santos, J.; Wofsy, S.C.; Trumbore, S.E. \& Martinelli, L.A. 2004. Forest structure and carbon dynamics in Amazonian tropical rain forests. Oecologia 140: 468-479. 